\title{
In Silico Taste - Toxicological Study of Chlorfenvinphos, Dichlofluanid, Fonofos, or Methacrifos Partial Degradation Products
}

\author{
Kafa Khalaf Hammud \\ Ministry of Science and Technology - Iraq
}

\section{Article information}

\section{Article history:}

Received: September, 21, 2021

Accepted: November, 12, 2021

Available online: December, 14, 2021

\section{Keywords:}

Chlorfenvinphos,

Dichlofluanid,

Fonofos,

Methacrifos,

Hypothetical degradation

*Corresponding Author:

Kafa Khalaf Hammud

kafaakhalaf@gmail.com

DOI:

https://doi.org/10.53523/ijoirVol8I3ID84

\begin{abstract}
Toxicity is important factor to human and environment and can be tested in lab and by computerized models. ProTox-II is in Silico method to assess safety of chemicals to minimize risk health threating to human and other living organisms in nature. Taste of material is another character can be calculated in Silico model like virtualtaste. Here, first attempt of using two computerized methods and hypothetical partial degradation products of four toxics materials used to control agricultural productivity was carried out to predicate taste and toxicity characters. LD50, Toxicity Class, organ and end point toxicities, Tox21-Nuclear receptor signaling and stress response pathways of Chlorfenvinphos, Dichlofluanid, Fonofos, and Methacrifos with their hypothetical degradation products were calculated. Hypothetical degradation products were a results of (C-C, C-O, C-N, C-S, C-P, P-O, P-S, or N-S) bond breakage. The hypothesized degradation chemicals showed that most of them were with sour taste and their toxicity were less class compared to the parent compound but not to non-toxic material (Class 6, LD50 more than $5000 \mathrm{mg} / \mathrm{kg}$ ). Also, they were structurally toxics and could be interact with molecular cellular target resulting than parent compound if they presented in required concentration.
\end{abstract}

\section{Introduction}

Toxicity is a major challenge to environment caused by industrial and urban contaminations that threats living things even at low concentration. Synthesized sulphur or phosphorous pesticide, insecticide, fungicide, acaricide, and other classes contribute in increasing health threating to human and other living organisms in nature. The persistence of these compounds is contamination source to food chain and health problem [1, 2]. In human or other exposed organism, these toxic substances have a potential damage for example on the nervous system which might lead to death beside mutagenic and carcinogenic effects. So, monitoring their degradation cycle and their persistence impact are necessary to control them in advance [3]. Nature has been exposed to broad spectrum of these toxic substances and one of them are Chlorfenvinphos, Dichlofluanid, Fonofos, and Methacrifos. 
These mentioned substances are classified as toxic to fish or bees and used as insecticide (Chlorfenvinphos, Fonofos, and Methacrifos) fungicide (Dichlofluanid), acaricide (Chlorfenvinphos and Methacrifos) with remarkable lethal dose (LD50) [4]. In 2007, a published article studied the degradation of 15 organophosphorus insecticides in three types of water sources under different laboratory and environmental conditions. The authors found that major factor influenced degradation process are temperature, $\mathrm{pH}$, oxygen, sunlight, oxygen, and type of water that present a varied half -live (4 days to 192 days) [5].

To eliminate or minimize the effects these toxics in guarantee way with economical safe path, many strategies like physical (adsorption), chemical (oxidation, photocatalysis, alkaline or acidic hydrolysis), thermal treatments but these treatments are costly and formed complexes as secondary pollutants. So, these incomplete treatments were replaced with environmentally - friendly - economical bioremediation strategy. But this strategy needs more studies of all these toxics from all points of view [6]. This need lights us to take part in this important issue by using software especially online type like http://tox-new.charite.de/protox II website to evaluate toxicity of hypothetical degradation of randomly selected toxics. ProTox-II is in Silico method to assess safety of chemicals to minimize risk especially in pharmaceuticals. This machine - learning predication model provides significant benefits may be obtained from in vitro and in vivo assays [7]. Hypothetical degradation products were a results of (C-C, C-O, C-N, C-S, C-P, P-O, P-S, or N-S) bond breakage. In this case, no chemicals, tools, instruments, money, health impact resulted from experiments will be presented. This website enables us and others to calculate many factors such as $\mathrm{LD}_{50}$ and toxicity, Hepatotoxicity, Carcinogenicity, Mutagenicity, Cytotoxicity, and others that indicate toxicity of the calculated molecule. The hypothesis reaction may be achieved by any above treatments.

In drug and food industry, taste is an important factor beside other basic issue. This factor can be predicted by in Silico method through http://virtualtaste.charite.de/VirtualTaste/ website. This predication may light us and others to probable use of new synthesized derivatives in food or drug industry. This online website gives three taste properties (Bitter, Sweet, and Sour) with predication probability (number with colour represents confidence of calculation) and predication strength (high, medium, and low) (Image 1.). According to the http://virtualtaste.charite.de/VirtualTaste/ home website "The taste of a chemical compound present in food stimulates us to take in nutrients and avoid poisons. Many active ingredients present in drugs taste bitter and thus are aversive to children as well as many adults. Bitterness of medicines presents compliance problems and early flagging of potential bitterness of a drug candidate may help its further development. Taste prediction of a compound is of large interest for the food industry". Image 1. give a good presentation of this in Silico method of known compound (acetic acid).

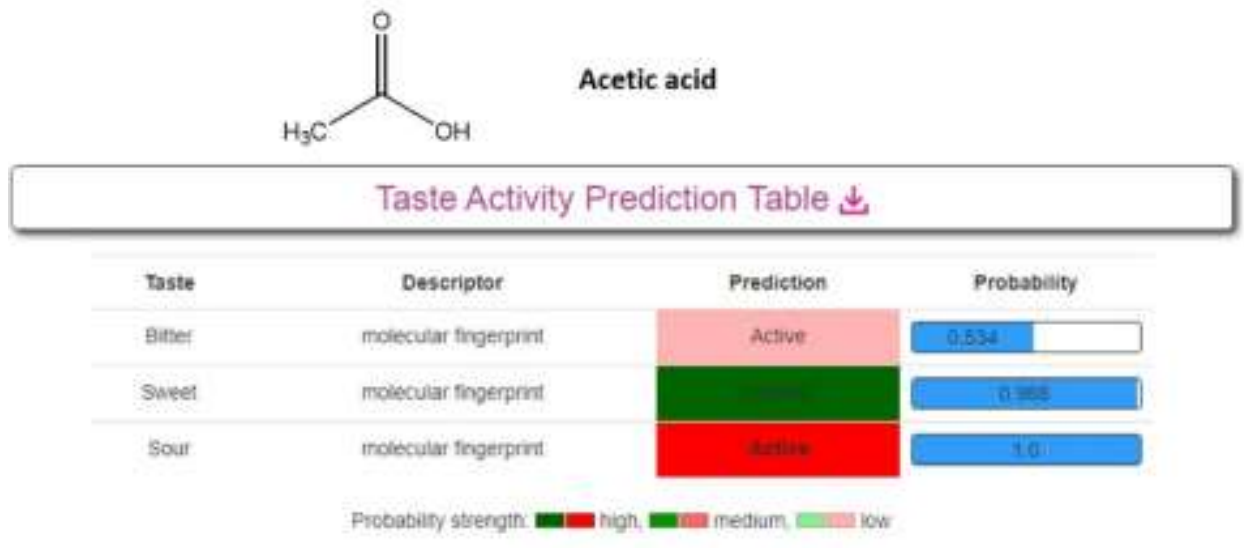

Image (1). Predication taste result of acetic acid by applying http://virtualtaste.charite.de/VirtualTaste/ website.

The purpose of this article and calculations is to lead the way to others especially in Iraq to do their calculations before involving in real experiments and estimate the toxicity to prevent it or reduce it to minimum numbers. 


\section{Experimental Procedure}

Hypothetical partial degradation: Chlorfenvinphos, Dichlofluanid, Fonofos, or Methacrifos were hypothetically subjected to partial degradation through different (C-C, C-O, C-N, C-S, C-P, P-O, P-S, or N-S) bond breakage (Schemes 1.\& 2.).

Calculations: Toxicological calculation of Chlorfenvinphos, Dichlofluanid, Fonofos, or Methacrifos and their hypothetical degradation products was predicated by http://tox-new.charite.de/protox II online website while taste calculation was predicated by http://virtualtaste.charite.de/VirtualTaste/ online website (Table 1.).

\section{Results and Discussion}

Schemes (1. \& 2.) show a hypothetical degradation of the chosen toxics (Chlorfenvinphos (C), Dichlofluanid (D), Fonofos (F), and Methacrifos (M)). This hypothetical reaction might be occurred by specific enzymes or chemical hydrolysis, oxidation, or other bond cleavage in aqueous medium. Bonds such as different $(\mathrm{C}-\mathrm{C}, \mathrm{C}-\mathrm{O}$, $\mathrm{C}-\mathrm{N}, \mathrm{C}-\mathrm{S}, \mathrm{C}-\mathrm{P}, \mathrm{P}-\mathrm{O}, \mathrm{P}-\mathrm{S}$, or $\mathrm{N}-\mathrm{S}$ ) were converted to the corresponding group such as $-\mathrm{OH},-\mathrm{SH},-\mathrm{NH},-\mathrm{NH}_{2}$.

Online website calculations that presented in Table 1. showed that numerical data of all calculated molecules were ranged as below:

Bitter taste: 0.576 -0.81, Sweet taste: $\mathbf{0 . 5 0 4 - 0 . 8 2 1}$, Sour taste: $\mathbf{0 . 5 - 0 . 9 9 4}, \mathrm{LD}_{50}(\mathrm{mg} / \mathrm{Kg})$ : 3-3460, toxicity: 1-5, Hepatotoxicity: 0.54-0.87, Carcinogenicity: 0.51-0.73, Immunotoxicity: 0.73-0.99, Mutagenicity:0.54-0.90, Cytotoxicity: 0.66-0.88, Aryl hydrocarbon Receptor (AhR): 0.65-1, Androgen Receptor (AR): 0.94-1, Androgen Receptor Ligand Binding Domain (AR-LBD): 0.95-0.99, Aromatase: 0.55-1, Estrogen Receptor Alpha (ER): 0.71-0.98, Estrogen Receptor Ligand Binding Domain (ER-LBD): 0.86-0.99, Peroxisome Proliferator Activated Receptor Gamma (PPAR-Gamma): 0.87-0.99, Nuclear factor (erythroid-derived 2)-like 2/antioxidant responsive element (nrf2/ARE): 0.5-1, Heat shock factor response element (HSE): 0.5-1, Mitochondrial Membrane Potential (MMP): 0.53-0.99, Phosphoprotein (Tumor Supressor) p53: 0.77-1, and ATPase family AAA domaincontaining protein 5 (ATAD5): $\mathbf{0 . 9 2 - 1 .}$

From Table 1. and Figure 1., sour taste property was in high values of most tested compounds while C1, M1, M2, M3 were with sweet taste and F2 and M were with bitter taste. D and its hypothetical degradation products were in sour taste this may reflect the influence of sulphur $(\mathrm{S}=\mathrm{O}, \mathrm{N}-\mathrm{S}, \mathrm{C}-\mathrm{S})$ and / or Carbon - halogen presence. Any material can be sensing with sweet, bitter, sour, salt, and other tastes by binding with specific cellular protein receptor type $\mathrm{G}$ after interaction with saliva in mouth (oral sensing). This ingested material -mammalian taste receptor binding enters several steps such as cell depolarization, neurotransmitter release, sensory propagation that translates by Central Nerve System (CNS). Sweet, sour, or bitter taste of these compounds is a result of this specific binding of $\mathrm{G}$ protein -coupled receptor expressed by their cells in palate epithelia and tongue related hydrogen bonding, heteroatoms presence, water solubility, and charge [8].

As stated to toxicity categories presented in http://tox-new.charite.de/protox II website and the obtained results, hypothetical degradation gave less toxic compounds compared to the parent compound except degradation of $\mathrm{C}$ to $\mathrm{C} 1$, D to D4, and M to M1 or M3 gave same category(Table 1., Figure 2.). These good results are a good sign towards process of degradation and get more non- or less toxic materials. Lethal dose $\left(\mathrm{LD}_{50}, \mathrm{mg} / \mathrm{Kg}\right) \mathrm{of}$ the tested parent toxics in [4] are (Chlorfenvinphos in rat=6.6 (iv), 9.60 (orally); Dichlofluanid orally in mice= 1250; or Methacrifos (in rats= 678)) and only calculated LD 50 of Chlorfenvinphos was nearly to documented data. 

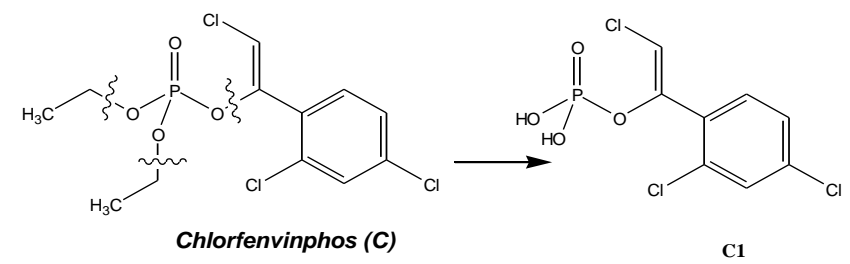

C1<smiles>O=C(CCl)c1ccc(Cl)cc1Cl</smiles>
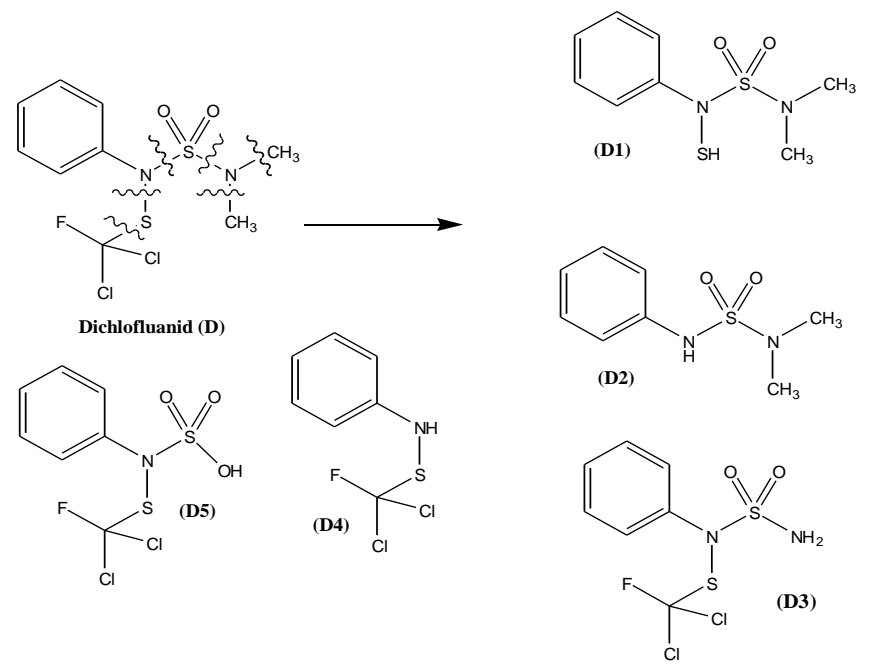

Scheme (1). Hypothetical degradation of Chlorfenvinphos or Dichlofluanid.

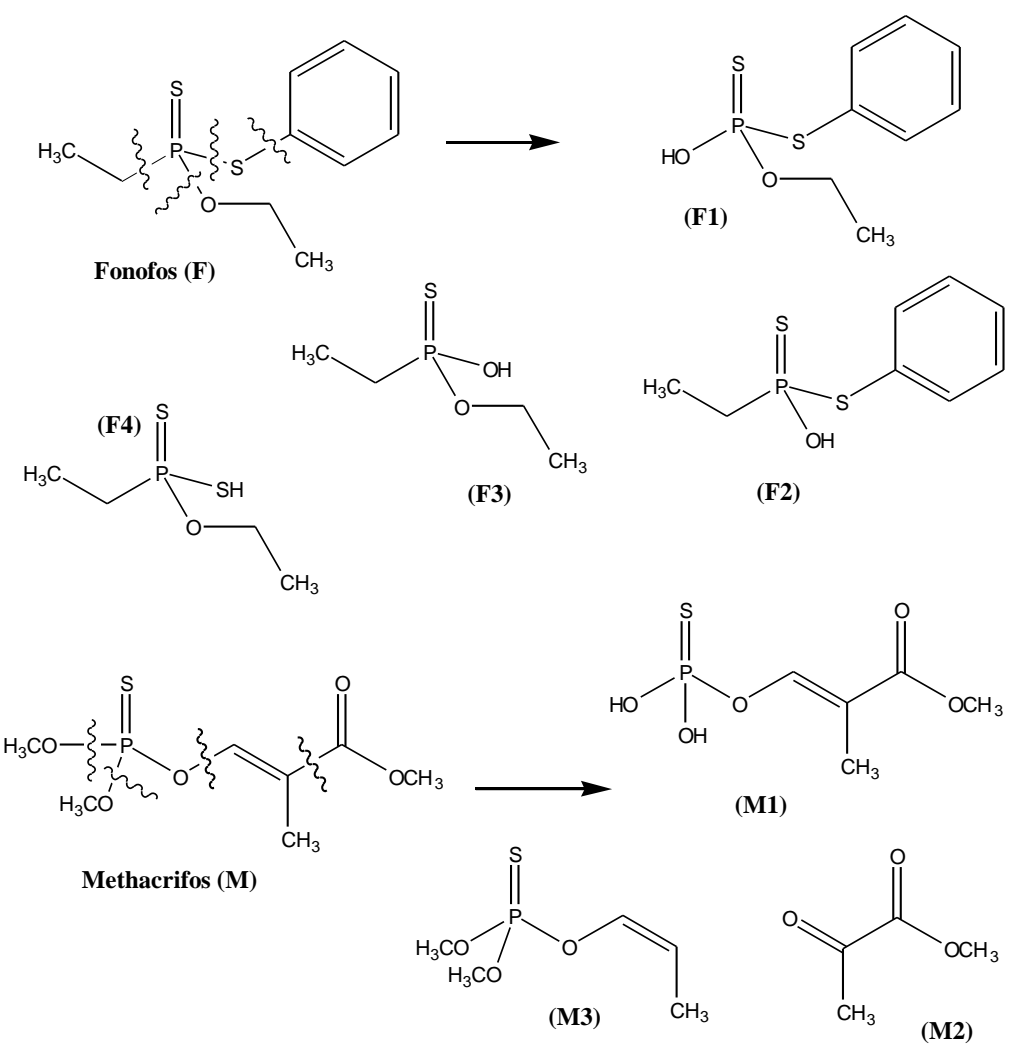

Scheme (2). Hypothetical degradation of Fonofos, or Methacrifos. 


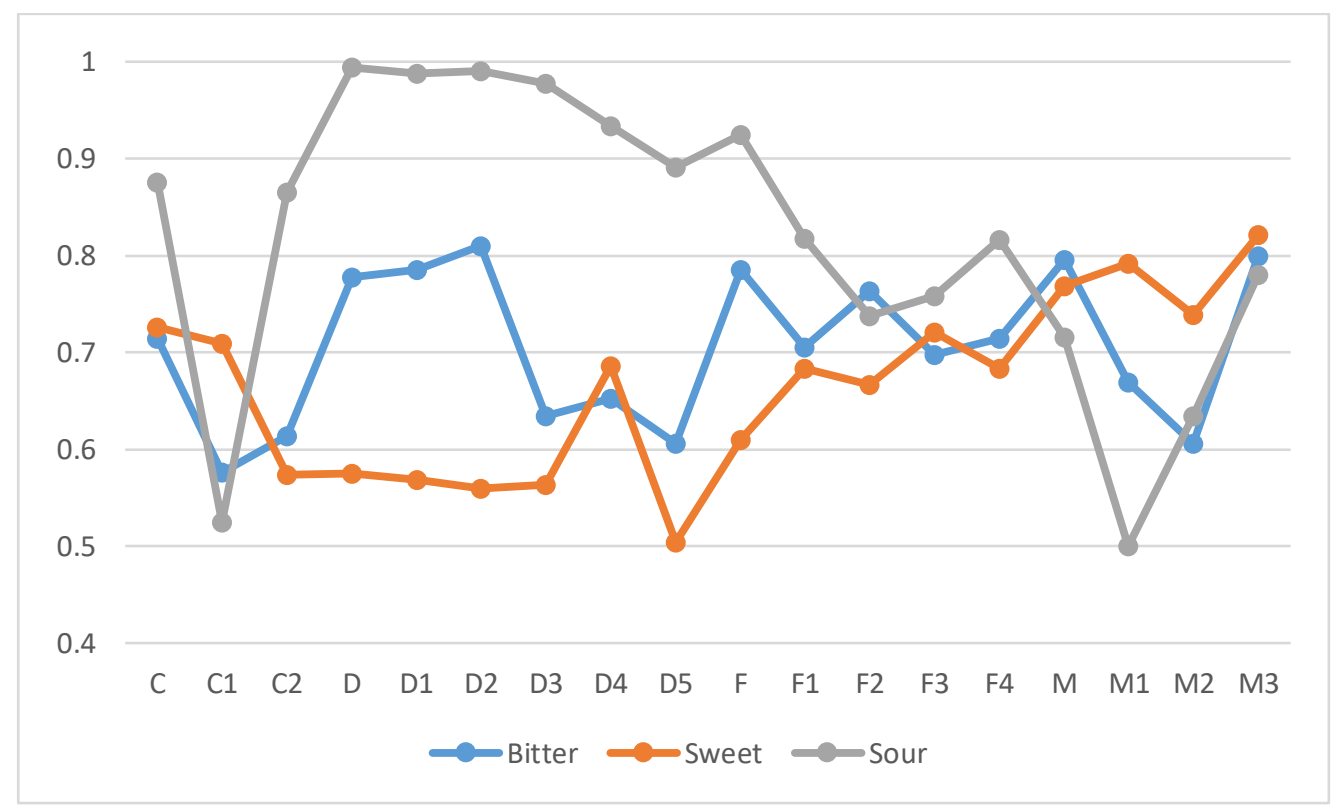

Figure (1). Taste predication of all tested compounds.

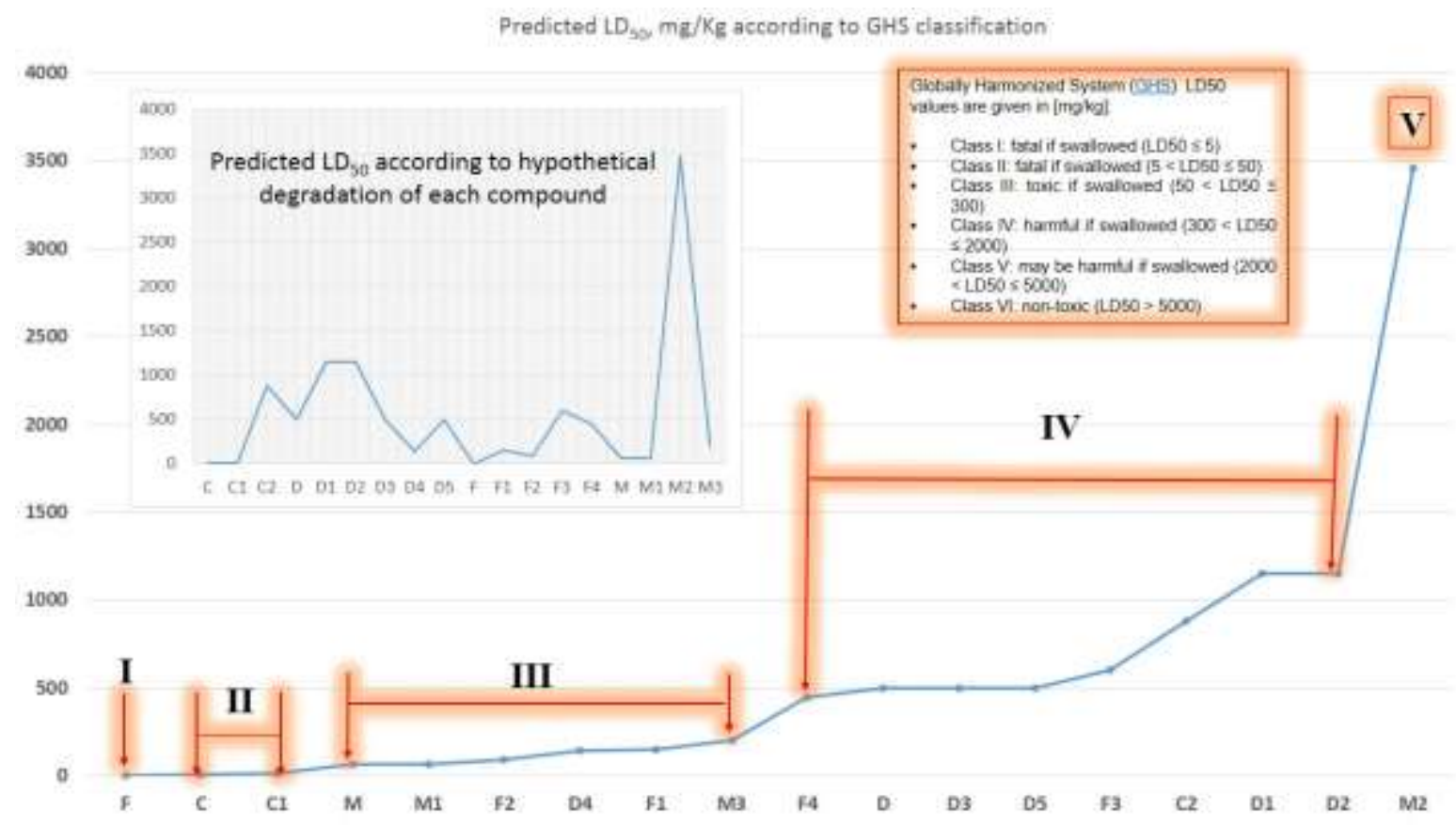

Figure (2). Predicated $\mathrm{LD}_{50}$ of the tested compounds.

Depending on Globally Harmonized System (GHS) toxicity classes, tested compounds showed that only F $\left(\mathrm{LD}_{50}=3 \mathrm{mg} / \mathrm{Kg}\right.$ ) was Class: 1 , fatal if swallowed with $\mathrm{LD}_{50}=5 \mathrm{mg} / \mathrm{Kg}$ or less while Class 2-fatal if swallowed $\left(\mathrm{LD}_{50}\right.$ not more than $50 \mathrm{mg} / \mathrm{Kg}$ ) was presented in $\mathrm{C} 1$ and $\mathrm{C} 2$; Class 3-toxic if swallowed (LD $\mathrm{L}_{50}$ not more 300 $\mathrm{mg} / \mathrm{Kg}$ ) in D4, F1, F2, M, M1, and M3; Class 4- harmful if swallowed (LD50 not more $2000 \mathrm{mg} / \mathrm{Kg}$ ) in C2, D, D1, D2, D3, D5, and Class 5- May be harmful if swallowed (LD50 less than $5000 \mathrm{mg} / \mathrm{Kg}$ ) in M2 (Table 1., Figure 2.).

Hepatotoxicity can be caused by chemical leads to acute liver failure. This sign is important especially in drug industry. This liver damage classification caused by increasing of special enzymes than the normal required level. Because there is no specific medication for it, chemical candidate as a drug must be identified to prevent 
this toxic from beaning a serious health problem $[9,10]$. It can be noticed that $\mathrm{D}$ and its hypothetical degradation products were with lowest Hepatotoxicity values while M3 have the highest value. In general, hypothetical degradation of the parent compounds $(\mathrm{C}, \mathrm{D}, \mathrm{F}$, and $\mathrm{M})$ may cause more liver damage and this is an important notice (Figure 3., Table 1.).

Inducing tumor can be a result of chemical or drug (carcinogen) with repeating dose for long time, accumulation, non- gastrointestinal adsorption, metabolism of a specific drug class, route and time of exposure ...etc. Carcinogenicity study needs long in - life of testing (rats, mice, then human) to be predicated with highly cost with is point online software may present an early choice of predication like http://tox-new.charite.de/protox II , https://preadmet.bmdrc.kr/toxicity/, or others $[11,12]$.

Carcinogenicity results of the tested compounds (Figure 3., Table 1.) were in less potential carcinogenic hazard from parent compound to its hypothetical degradation products except $\mathrm{C}$ (non-significant increase).

The other toxicological endpoint is Immunotoxicity that predicts industrial chemicals or drugs effect on immune system via in Vivo, in Vitro, and / or in Silico methods [13]. Immune functions can be affected by any compound according to body weight, food, lymphoid activity and other cellularities [14]. http://tox-new.charite.de/protox II website is easy to - use computational approach was used to screen out our hypothetical entities and showed that immune system is with high risk with these chemicals (Figure 3., Table 1.).

Mutagenicity is the term of human and other environmental species exposure to hazardous effect of chemical. This cytogenetic damage is consequence of heritable change in DNA. Many studies screened nucleotide sequence mutation by in vivo to secure quality assurance of chemicals $[15,16]$. The tested chemicals were in mutagenicity increase from parent compounds $(\mathrm{C}$ and $\mathrm{M})$ to their degradations while (D and $\mathrm{F}$ ) degradation products were in less cytogenetic character (Figure 3., Table 1.).

Cytotoxicity or the toxicity term of in vitro biocompatibility of chemical using primary cell as itself or in lines can be characterized from variation of cell functions or properties [17]. Cytotoxicity character of the tested compounds varied in their response to http://tox-new.charite.de/protox_II website calculation where most of them showed an increase compared to their parent compound as shown in Figure 3., Table 1.

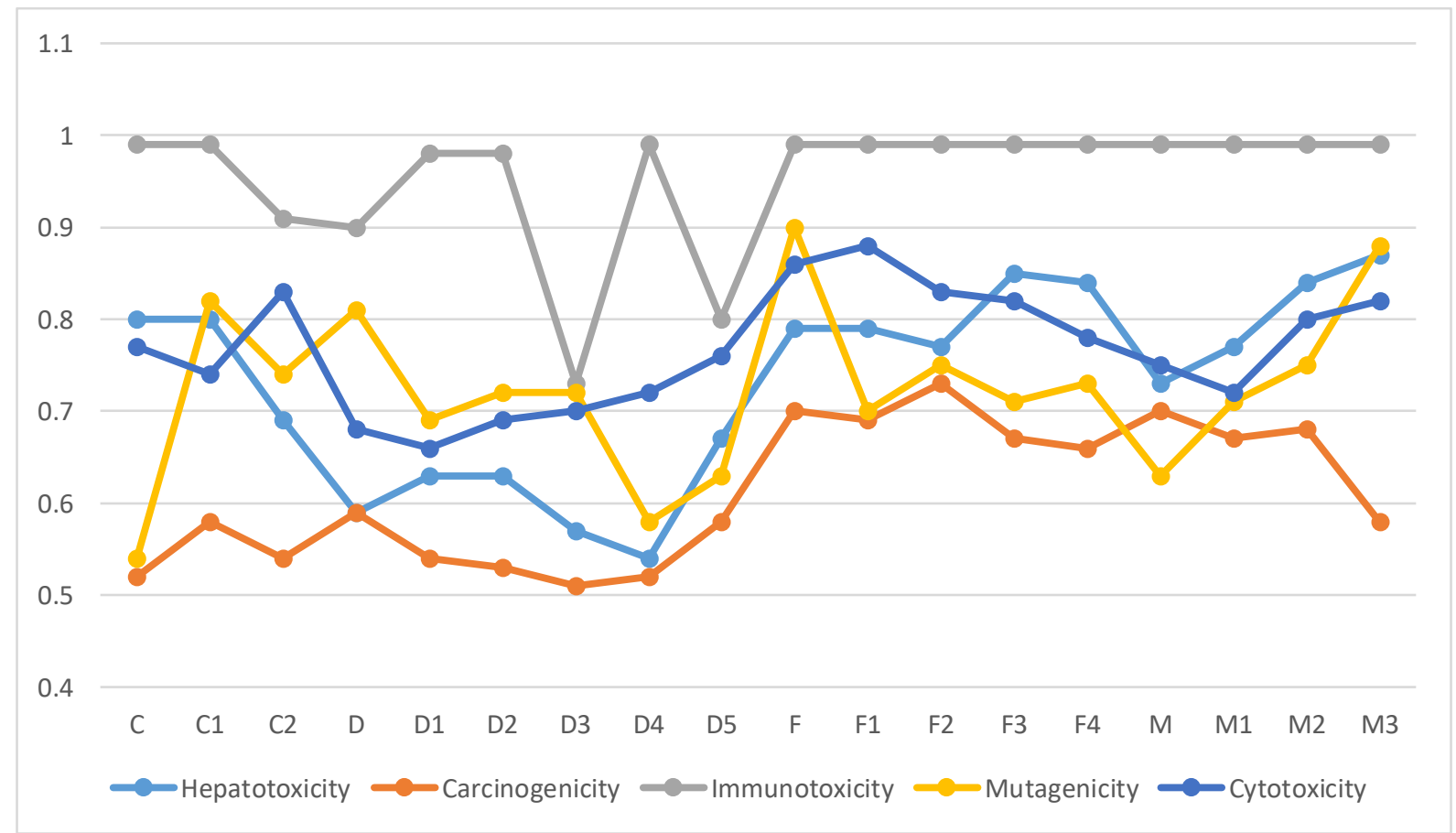

Figure (3). Hepatotoxicity, Carcinogenicity, Immunotoxicity, Mutagenicity, Cytotoxicity of the tested compounds. 


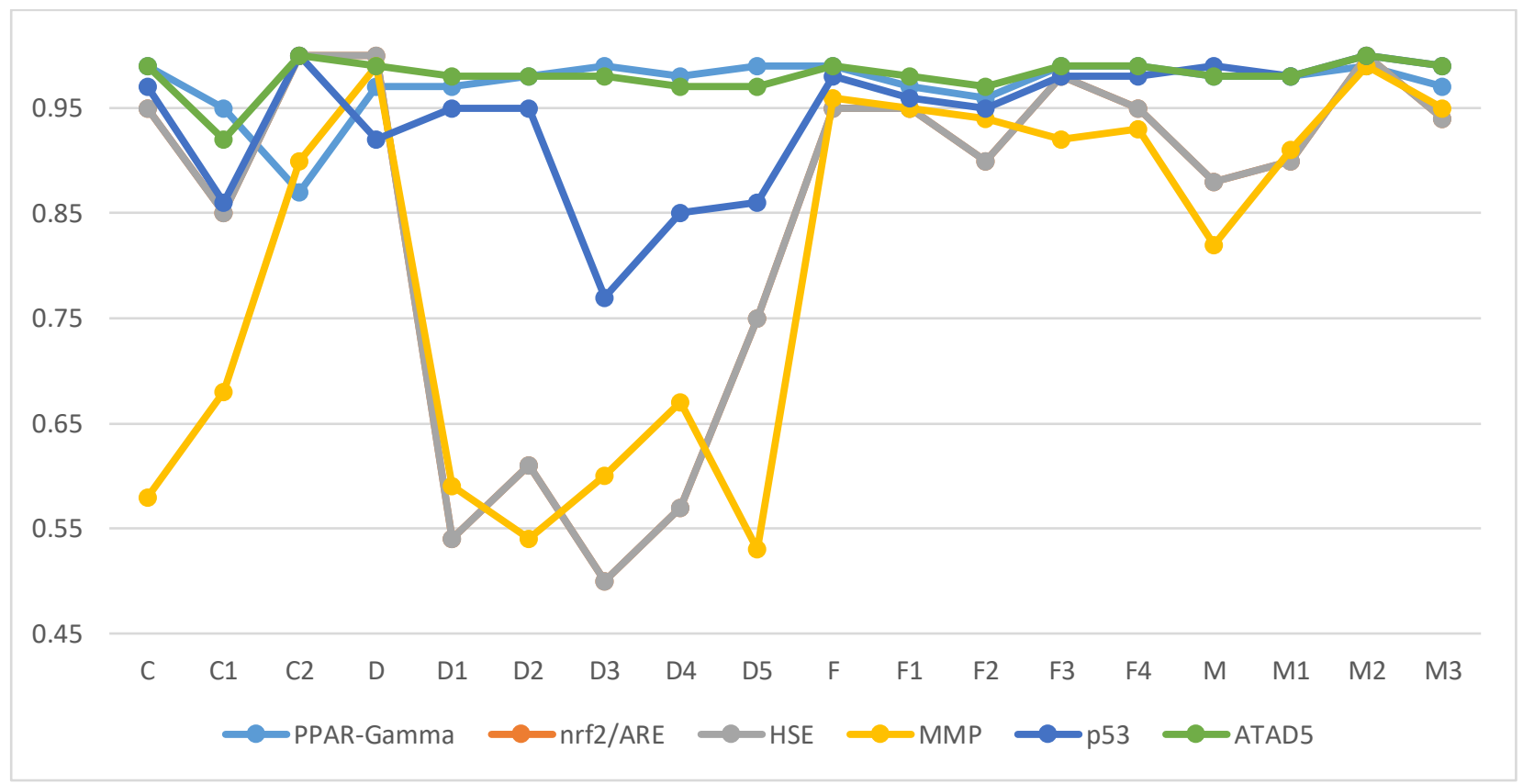

Figure (4). Target nuclear receptor signaling and stress response pathways predication.

Aryl hydrocarbon Receptor (AhR), Androgen Receptor (AR), Androgen Receptor Ligand Binding Domain (ARLBD), Aromatase, Estrogen Receptor Alpha (ER), Estrogen Receptor Ligand Binding Domain (ER-LBD), Peroxisome Proliferator Activated Receptor Gamma (PPAR-Gamma), Nuclear factor (erythroid-derived 2)-like 2/antioxidant responsive element (nrf2/ARE), Heat shock factor response element (HSE), Mitochondrial Membrane Potential (MMP), Phosphoprotein (Tumor Supressor) p53, and ATPase family AAA domaincontaining protein 5 (ATAD5) were predicated with http://tox-new.charite.de/protox II website. In general, these target nuclear receptor signalling and stress response pathways characters showed random response from parent compounds $(\mathrm{C}, \mathrm{D}, \mathrm{F}$, and $\mathrm{M})$ to their hypothetical degradation products with mainly high values as noticed in Figure 4. and Table 1.

Now to understand our calculations, there is a comparison between Chlorfenvinphos (C) and one of its hypothetical degradation product (C2) (Figure 5, Table 1.). First, Chlorfenvinphos (C) predication by proTox-II showed it as a fatal if swelled with $\mathrm{LD}_{50}$ value $10 \mathrm{mg} / \mathrm{Kg}$ as well high confidence score related to five toxicological pathways (AhR, AR, AR-LBD, Aromatase, and ER). Also, toxicity target predicators of C were with highly probable binding to Androgen and Progesterone and this predication harmonized with its toxicity class 2. Additionally, high hepatotoxicity predication value worked with stress response predication. So, this Chlorfenvinphos compound is structurally toxic compound and may interact with molecular target in cell or organ resulting toxic response.

The second hypothetical degradation product of Chlorfenvinphos (C2) presented less toxicity class (4) with high $\mathrm{LD}_{50}$ value $880 \mathrm{mg} / \mathrm{Kg}$ compared to $\mathrm{C}$. C2 was with high confidence score of toxicological pathways but less than $\mathrm{C}$ except AhR and AR. Highly probably binding of $\mathrm{C} 2$ to Progesterone and Androgen were less than the parent compound (C). Also, stress response predictors (nrf2/ARE, (HSE), MMP, p53, and ATAD5) of C2 were higher than C. In conclusion, C2 is structurally toxic compound and may be highly interactive to molecular cellular targets revealing toxic response than $\mathrm{C}$ if presented in required concentration.

In the same manner other parent compounds may be compared with their hypothetical degradation products to determine all toxicity predictors. 
Table (1). Taste - Toxicological data Chlorfenvinphos, Dichlofluanid, Fonofos, and Methacrifos with their hypothetical degradation products.

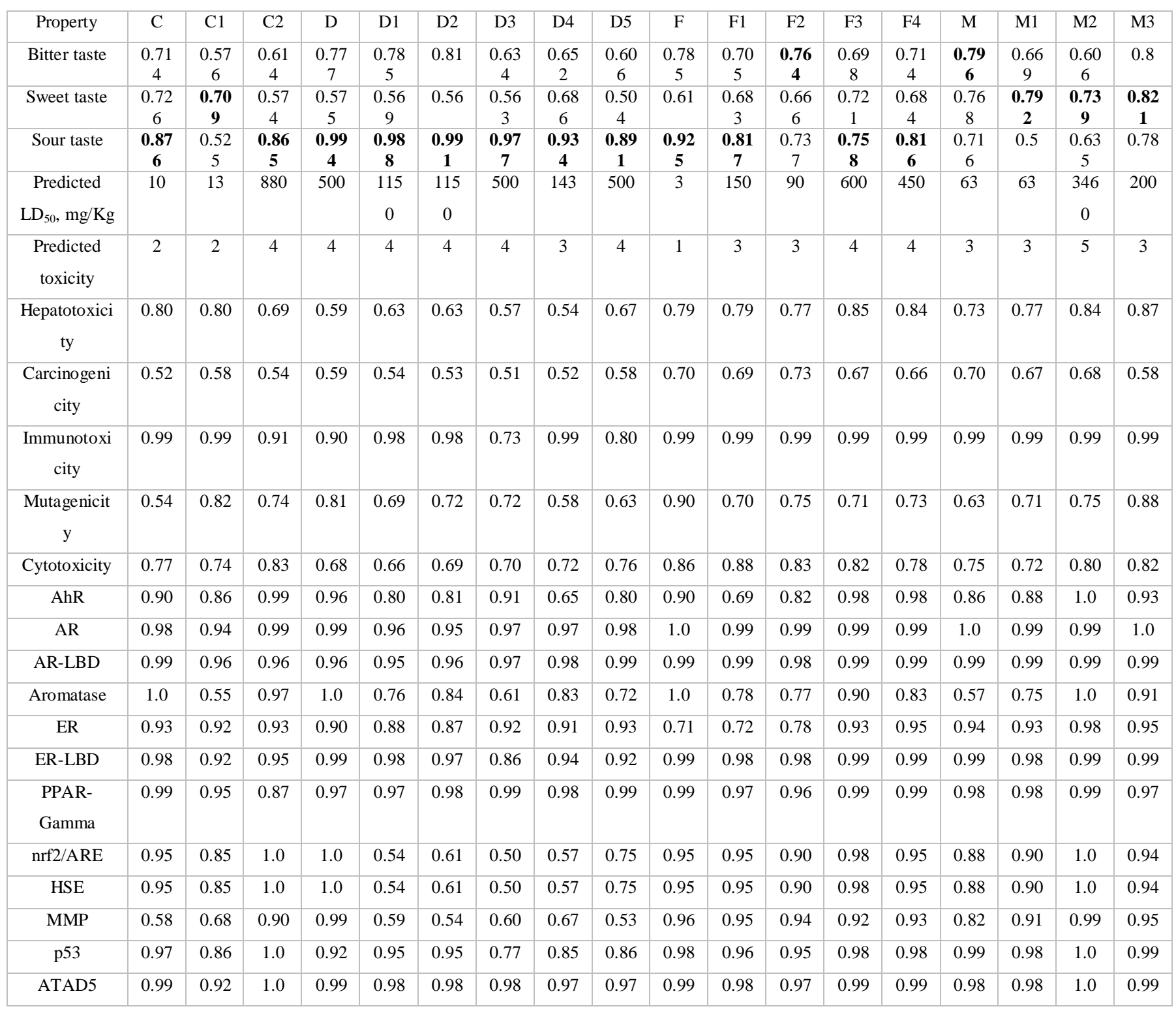

\section{Conclusion}

First attempt of using two computerized methods and hypothetical partial degradation products of four toxics materials used to control agricultural productivity was carried out to predicate taste and toxicity characters. According to calculations, most of products were with sour taste. Additionally, most of them were structurally toxic compound and may be highly interactive to molecular cellular targets revealing toxic response than parent compound if they presented in required concentration.

\section{References}

[1] A. Özkara, D. Akyil, and M. Konuk, "Pesticides, environmental pollution and health, environmental health risk-hazardous factors to living species," M. Larramendy and S. Sononeski (Ed.), IntechOpen, UK, 2016.

[2] M. de Araứjo, C. de Assis, R. de Sà, J. da Silva, B. de Veras et al., "Impact of agricultural toxicity on nontarget organisms in aquatic ecosystem, emerging contaminants," A. Nuro (Ed.) IntechOpen, UK, 2020.

[3] T. Rasheed, M. Bilal, F. Nabeel, M. Adeel, and H. Iqbal, "Environmentally - related contaminants of high concern: potential sources and analytical modalities for detection, quantification, and treatment," Environment International, vol. 122, pp. 52-66, 2019. 
[4] The Merck Index, "An Encyclopaedia of Chemicals, Drugs, and Biologicals," M. O' Neal, A. Smith, P. Heckelman, and J. Kinneary (Ed.), Merck \& Co., New Jersey, USA, 2013.

[5] B. Druzina and M. Stegu, "Degradation study of selected organophosphorus insecticides in natural waters." International Journal of Environmental Analytical Chemistry, vol. 87, no. 15, pp. 1079-1093, 2007.

[6] M. Ortiz-Hernández, E. Sánchez-Salinas, E. Dantán-González, and M. Castrejón-Godinez, "Pesticide Biodegradation: Mechanisms, genetics and strategies to enhance the process, Biodegradation-life science," R. Chamy and F. Rosenkranz (Ed.), IntechOpen, UK, 2013.

[7] P. Banerjee, A. Eckert, A. Schrey, and R. Preissner, "ProTox-II: a websever for the predication of toxicity of chemicals," Nucleic Acids Research, vol. 46, pp. W257-W263, 2018.

[8] J. Boughter and S. Munger, "Taste receptors in Encyclopaedia of biological chemistry," W. Lennarz and M. Land (Editors), $2^{\text {nd }}$ edition, Academic Press, ScienceDirect, Elsevier, USA, 2013.

[9] A. Paniagua and P. Amariles, "Hepatotoxicity by drugs, pharmacokinetics and adverse effects of drugsmechanism and risk factors," N. Malangu (Ed.), IntechOpen, UK, 2017.

[10] E. Biömsson, "Hepatotoxicity by drugs: the most common implicated agents," International Journal of Molecular Sciences, vol. 17, no. 2, pp. 224, 2016.

[11] K. Hentz, "Toxicology testing and evaluation, in Comprehensive Toxicology," ScienceDirect, Elsevier, USA, 2010.

[12] R. Benigni and C. Bossa, "Mechanisms of chemical carcinogenicity and mutagenuicity: A review with implications for predicative toxicology," Chemical Review, vol. 111, no. 4, pp. 2507-2536, 2011.

[13] A. Schrey, J. Seeber, M. Drwal, P. Zwicker, N. Schultze, et al., "Computational predication of immune cell cytotoxicity," Food and Chemical Toxicology, vol. 107, Part A, pp. 150-166, 2017.

[14] I. Hueza, P. Raspantini, L. Raspantini, A. Latorre, and S. Górniak, "Zearalenone, an estrogenic mycotoxin, is an immunotoxic compound", Toxins, vol. 6, no.3, pp. 1080-1095, 2014.

[15] H. Du, B. Pan, and T. Chen, "Evaluation of chemical mutagenicity using next generation sequencing: A review," Journal of Environmental Science and Health, Part C, vol. 35, no. 3, pp. 140-158, 2017.

[16] K. Rim and S. Kim," A review of mutagenicity testing for hazard classification of chemicals at work: focusing on in vivo micronucleus test for allyl chloride," Safety and Health at Work, vol. 6, no. 3, pp. 184191, 2015.

[17] B. David, M. Jane, and C. Marco, "The natural cytotoxicity receptors in health and disease," Frontiers in Immunology, vol.10, pp. 909, 2019. 